\title{
ON THE DETECTION OF OPEN AND SHORT FAULT IN SWITCHED RELUCTANCE MOTOR WITH CLASSIFICATION
}

\author{
${ }^{1}$ Chandrika, V.S. and ${ }^{2}$ A. Ebenezer Jeyakumar \\ ${ }^{1}$ Department of EEE, P.S.V. College of Engineering and Technology, Krishnagiri, India \\ ${ }^{2}$ Sri Ramakrishna Engineering College, Coimbatore, India
}

Received 2013-07-24; Revised 2014-01-12; Accepted 2014-01-23

\begin{abstract}
Switched reluctance motors are tremendously increasing in usage. Monitoring the motor performance online is essential, in order to maintain the health of the motor as well as for a continual application process carried out by the SRM. Though certain fault detection methods are available based on comparator circuits, those methods are inefficient, because they need human attention to identify the faults. In this study, we propose a wavelet based SRM power drive fault detection and classification using BPN method. The currents flowing in different phases and the known states of all power switches helps us to find out the magnitude of the converter supply current, since an asymmetric bridge converter is used. In this study, short and open circuit fault occurrences in the converter power switches are presented for various coils of the stator windings. The proposed technique is able to detect these faults and identify the affected stator phase as well as the faulty arm along with the the time instant of fault occurrence.
\end{abstract}

Keywords: Discrete Wavelet Transforms (DWT), Back Propagation Network (BPN), Switched Reluctance Motor (SRM)

\section{INTRODUCTION}

Usually faults in motors results in the less life time of the motor. These faults may occur in the motor side or at the power drive section. Generally speaking, in any circuit, a short circuit is defined as an infinite electric current in a closed path which may even spoil the path of the current flow due to the rise in the temperature in the closed path. When the same scenario is considered in a motor, this may lead to disconnection of the motor phase. This disconnection may occur due to physical damage of conductor or due to the trip circuit installed in the circuit. Once a short circuit fault occurs, there is a chance that it is not caught in observations, because of low speed operations or at partial load conditions. At these conditions, the phase current may not exceed and reach the magnitude of the short circuit current and hence a sudden failure may not occur. But this can not be taken as advantage because such faults may eventually reduce the motor life time. So such faults should viewed Corresponding Author: Chandrika, V.S., Department of EEE, P.S.V. College of Engineering and Technology, Krishnagiri, India

seriously. Early detection will always help to decide appropriate actions of whether to stop the switching pulses to the particlar phase or to eliminate the phase itself. The salient pole configuration of SRM produces ripples in torque especially at high speeds. Various methods to control the torque ripples have been proposed in earlier literatures to maintain a smooth operation. But at abrupt faulty conditions, if the motor phase is disconnected, then it may lead to the deterioration of electromagnetic and mechanical behavior. Ultimately the rated power decreases and introduces an unbalance in the rotor forces. Such disconnection of phase from the circuit may be due to the converter faults or due to the motor itself. Or, faults in power converters may lead to the physical damage of motor phases, vice versa. At this situations it is wise to implement a strategy to detect the faults to save the motor and to increase the motor life time. By common sense, we know that the less reliable part in the over all drive system is the power converter. Though there exists, Fuzzy based method as in

84 JCS 
(Mohanraj et al., 2012), it decides the faults based on the RMS current values. But in real time mechanically loaded conditions, these currents may vary based on the load. Hence fuzzy based approach may not be suitable for detecting faults just only with RMS values of the currents. More over instant of fault occurrence can not be found with this fuzzy based approach. Since, a rule based fault detection is done, speed of detecting the fault is better in fuzzy based systems. Kohen's self organizing map technique has been used in (Jaganathan et al., 2011). This method uses $i_{d}$ and $i_{q}$ to estimate the performance. It is obvious that it takes about 500 epochs as given in (Jaganathan et al., 2011). The data base involved in the estimation process is very huge and time consuming. So earlier detection is not possible in these methods. Also, time localization has not been addressed in (Jaganathan et al., 2011). Even in (Salankayana et al., 2012), neural network based approach is used, where RBF training has been done with 7000 neurons as inputs. Instant of fault occurrence is not detected and takes huge training time. A maintenance free Switched Reluctance Generator has been proposed in (Karthikeyan et al., 2010), where transient analysis of flux linkages and Inductance profile has been analyzed. Various control strategies have been reviewed in (Susitra et al., 2010), with lack of fault detection schemes in SRM. A difference in the Dc current value is used to detect the open and short circuit fault detection in (Gameiro and Cardoso, 2012). Though this is a direct method, it is really crucial to follow the current waveforms and its behavior at mechanically loaded conditions. Hence, in this study, a wavelet based decomposition has been used to detect the short and open circuit faults in a power switch which is the main reason for a faulty operation. After the detection of the fault, a classification is done using neural network based approach, to find the phase of the fault occurred windings or switch. A faultdetectable power converter, can be used to almost get back the healthy operation. This study has been organized as follows. Section 2 deals with the healthy motoring operation, section 3 discuss about the Faulty motoring conditions, section 4 gives a summary of wavelet transforms and classification techniques, section 5 elaborates the proposed method and section 6 shows the simulation outputs.

\section{MOTORING OPEARTION (NORMAL)}

In SRM, the inductance profiles depends on the rotor position. This is mainly due to the double salient pole configuration. Figure 1 shows an 8/6 SRM.

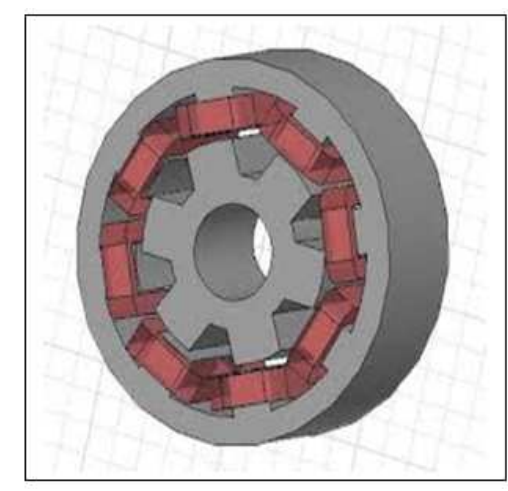

Fig. 1. 8/6 SRM

In SRM, when a particlar phase current increases, it leads to a saturation and hence there exists a decrease in the inductance amplitude. This sounds more when the rotor and stator pole are aligned together. The saturation effect is more at these perfectly aligned positions. No saturation occurs at un aligned positions of the poles (when two rotor poles are at same distance from the stator pole). Two torques are produced in SRM while a supply is applied to a corresponding phase. When the rotor pole is approacing the stator pole, an electromagnetic torque is created to maintain the alignment of the poles. This is motoring action. In case, if the stator and rotor poles are moving away from each other, then it produces a braking torque. To avoid unnecessary negative torques in motoring mode, each phase is conducting between aligned and unaligned positions. While analysing the electromagnetic behaviour, it is good to analyse individual phases seperately. Because interactions are less between the phases and hence phase to phase analysis is not needed. More over it is possible to neglect the mutal inductances because of its least magnitudes. In order to operate the individual phases independently the control pulses are applied independently. By using asymmetric bridge converter we are able to achieve this. Hence this type of converter is very popular in SRM. There are two transistor switches and two diodes are connected to each phase (Gameiro and Cardoso, 2012).

\section{OPEARTION AT FAULTS}

It is essential to discuss the operation of the SRM drive in order to analyse its fault conditions. The topology shown in Fig. 2 consists of 8 switches and 8 free wheeling diodes. In order to energize a phase, two 
switches corresponding to a phase is turned ON, in such case, the source voltage (+Vs) appears across the phase ' $a$ ' coil which constitutes a phase current. While the machine is in motoring mode and the phase is conducting, through only one switch, the motor is energized mechanically from magnetic energy. At this instance, there is no current flow from source to the stator phase. When both the switches are switched on and the phase current flows, some amount of power is returned back to the source and some amount of power is transferred into mechanical energy. Which means, the particular phase of the SRM acts as a generator. The switches are designated as $S_{U n}$ and $S_{L n}$ to differentiate upper and lower positions. The desigations $S_{U a}, S_{U b}, S_{U c}, S_{U d}$ represents upper switches of phase a, b, c and d respectively. The designations $\mathrm{S}_{\mathrm{La}}, \mathrm{S}_{\mathrm{Lb}}, \mathrm{S}_{\mathrm{Lc}}, \mathrm{S}_{\mathrm{Ld}}$ represents lower switches of phase a, b, c and d respectively as shown in Fig. 2. The switching are well shown in (Gameiro and Cardoso, 2012) for healthy operation.

In SRM, the inductance of the coil phases depends on the rotor position is a known fact. The related phase current beaviour increases with an increase in the motor speed. Hence the methods to control the speed can not be same for higher and lower speeds. This is because, the back EMF increases with increase with speed. The situations are analysed distinctly at low speed and high speeds. In motoring mode, at lower speeds, the phase current increases when a power supply is applied between the phases i.e. when $S_{U a}$ and $S_{\mathrm{La}}$ are switced on. At higher speeds, this increase occurs until the back EMF becomes higher than the applied voltage when the rotor and stator poles overlap. At such working conditions, ignition angle and commutation angle are the control paramaters where the phase starts to magnetize and both switches $\mathrm{S}_{\mathrm{Ua}}$ and $\mathrm{S}_{\mathrm{La}}$ are turned off respectively. At lower speed operations, phase current is supplied between ignition and commutation angle to maintain appropriate current. In soft chopping based gate pulse generations, one switch is permanently switched on and the other is switched between on and off states to regulate the phase current. In SRM the major task is to minimize the ripples in torque especially at high speeds. Control strategies are well discussed in (Paramasivam and Arumugam, 2004). But unfortunately these methods are meant for lower operating speeds. The above discussion is meant for a normal motoring operation. An open fault in an SRM disconnects a particular phase. A short circuit fault makes a current flow higher than it is needed to maintain particular magnetization and restricts fast demagnitization, in other words to say the flux saturates. Both the fault cases are to be dealt seriously, hence in our work we detect both short and open circuit faults. At certain swtiching conditions, it is not possible to detect faults, because, the faults do not produce a variation in current flow, because the faulted switch operates as it is expected to do so (Gameiro and Cardoso, 2012). When there exists an appreciable change in current flow, it would be easy to detect the fault. Such un expected current flows can be detected using wavelet transforms which would be better than any electronic circuitry. Electronic circuit is not capable of recording the time instant of fault occurrence is its main draw back. In high end research works, we would need the instant of fault occurrence to trace back the associated circuits and control elements in an over all drive system. Hence with the help of detail coefficients obtained there is a key to find the instant of fault occurrence.

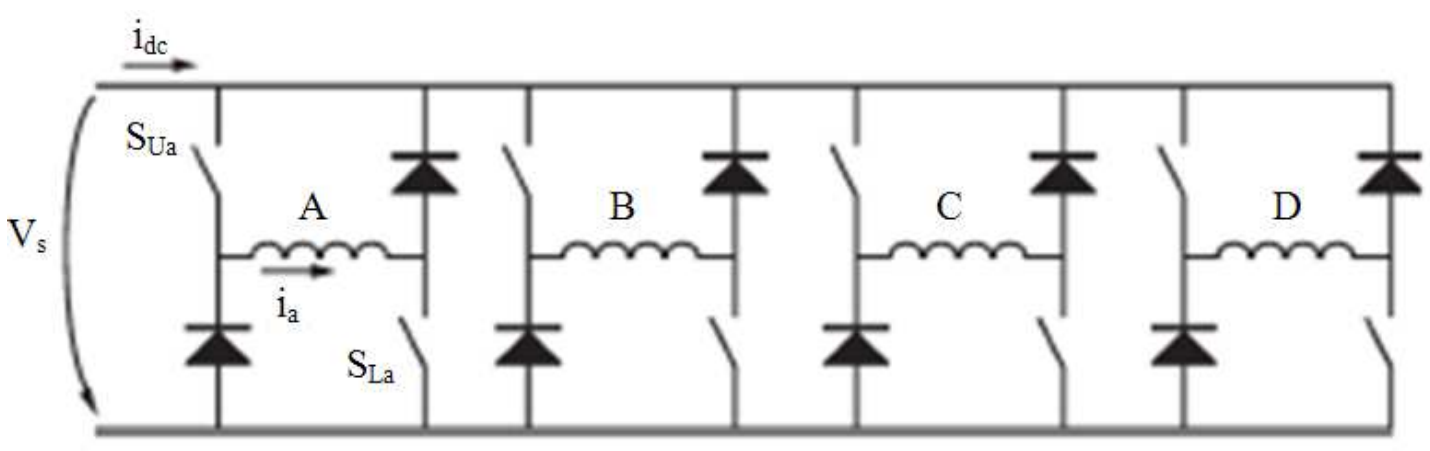

Fig. 2. Power converter for 4 phase SRM 


\section{DISCRETE WAVELET TRANSFORMS}

Wavelet transform is a good method to decompose a time domain signal into its high frequecy and low frequency components. This is well suited to analyse time domain signals with discontinuity. Wavelets have a property to detect such discontinuity in signals and sudden changes in amplitudes as well. A time domain signal when transformed in wavelet domain, it is shown with respect to amplitude, time and frequency. In fourier transform, only the amplitude and frequency relationships are obtained. The time domain signal considered is expressed as a set of functions defined by orthogonal functions called as wavelets. Mother wavelets can be considered of any type like Haar, B-spline, Coiflet, Daubechies.

\subsection{Time Localization}

The conditions of the motor has to be monitored continuously in order to verify the integrity of the motor. In addition to the detection of the fault, it is needed to find the time instant of the fault. It may be thought why to find out the time instant and it is enough to detect the fault alone and disconnect the phase in such cases, but at high end research works, a back end analysis is needed and it is essential to find the time instant of fault to track the associated circuits is our best justification for the need of time localization of fault occurrence. From mathematical point of view, spectral components of the time domain signal can be viewed from stator phases. Voltage, current and power may be used to find out turn faults, brokern rotors, bearing failures and air gap eccentricities.

\subsection{D Wavelet Transform}

The wavelets are nothing but a short duration energy signal. Any signal which satisfies the two properties may be considered as wavelets. They are 1 . Mean value of the short duration signal is zero and 2 . The energy value of the signal is finite. The signals which we take into account in our works are basically one dimensional signals. Hence, we need to address only 1D wavelet transforms. Applications of wavelets are enormous and even applied in image processing where 2D wavelets are used as given in (Shanmugam et al., 2011). Any how analysing 2D wavelets is not essential in our proposed work. After applying wavelet transforms, the time domain signal is transformed into wavelet coefficients and these coefficients are associated with two variables namely dilation and translation. Transalation means, the time and Scaling means the method of viewing frequency. When the scale is smaller, it means that high frequency content of the original signal is analysed. When the scale is larger, it means that the low frequency content is viewed. The test waveforms which we consider for the open and short faults are typically non periodic signals which contains high frequency oscillations and localized impulses. While using Discrete Fourier Transform (DFT) this creates a problem because DFT is associated with periodic signals. In power electronics and drives, the DFT alone is not sufficient to track the faults. When we make an attempt to alter the signal in localized time instant, the whole spectrum may be affected. In order to reduce such effects, wavelet transforms are preferred, where both time and freuquency analysis can be made. It may be appropriate to use Short Time Fourier Transform (STFT) also but, it is limited to a fixed window size. Hence the resolution in such analysis will be kept fixed in the resulting transform. But in case of wavelets we may be able to focus on short time intervals for high frequency components and long intervals for low frequency components. This enhances the results of analysis with localized impulses and oscillations.

\subsection{Classification}

The classification of faults are done based on supervised learning method. In neural networks decision may be taken based on supervised and unsupervised methods. Wavelet coefficients obtained from SRM electrical waveforms are used as training inputs. With these known values of the targets, the neural network is trained. During training session, the weight vectors are adjusted in such a way to reach te desired target output. Once the error goal is reached, the training process is stopped. This training is done with wavelet coefficients obtained at both healthy and faulty conditions. During the testing phase, at online, while the motor is running, these wavelet coefficients are applied as test inputs to the neural network. The precalculated weights are used now to detect the target value. Based on the type of fault the output neuron outputs a ' 1 '. In our case the number of inputs to the neural network being 1 neuron at input layer and 3 neurons at the output layer. Number of neurons in the hidden layer is kept equal or more than the input layer. More number of neurons in hidden layer will give higher accuracy and takes more time for training as well as testing.

As shown in Fig. 3, the general architecture of BPN, the input layer is connected to the hidden layer and the hidden layer is connected through a multiplication factor by weights. 


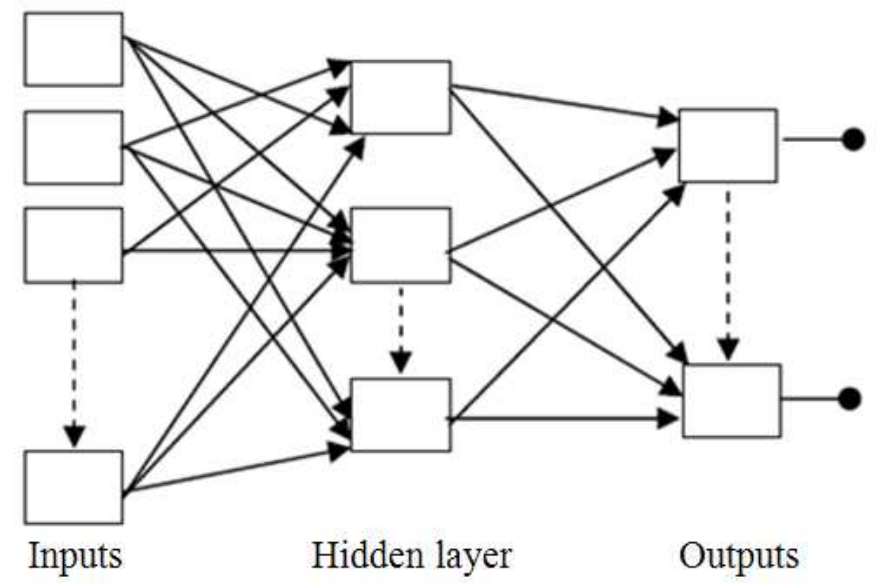

Fig. 3. Architecture of BPN

The training algorithm consists of 4 phases namely:

- Weights initialization

- Forward propagation

- Back propagation

- Weight updating process

The training process is carried out based on two criteria, either the number of epochs or the reach of the minimum error goal. For each epoch, the output value is calculated and the difference between the target and the obtained value is the error obtained in that particular epoch. This error is again back propagated into the network. Doing so, the network adjusts by itself, its weights in such a way the error is minimized. When the minimum error goal is reached, the iterations are stopped and training process is assumed over. It is always suggested to keep the error goal to be very minimum in order to get good accuracy in classification. But the least error target will increase the training time, but need not be worried because training is done once and it is done at off line. So it no way affects the dynamic test conditions and time consumption. A method of using neural network for fault detection approach is discussed and implemented in (Sivakumar and Parvathi, 2013).

\section{PROPOSED FAULT DETECTION METHOD}

An SRM of $8 / 6$ available in MATLAB library is used to design the simulink model is run at steady state at $2000 \mathrm{rpm}$. The simulation model is shown in Fig. 4. The simulation model consists of a PID controller in order to maintain the set speed in a closed loop system. Where ever needed, the values of currents are observed in MATLAB workspace using simout blocks. These 1D current values are applied simultaneously to the wavelet transforms and neural network algorithm, where we detect the faults and classify respectively. The mother wavelet of our choice is db1 (Daubechies wavelet). The sequence of mathematical operation in wavelet transform is given in the following discussion. The original signal $x[n]$ is passed through a high pass filter and low pass filter with transfer functions $\mathrm{g}[\mathrm{n}]$ and $\mathrm{h}[\mathrm{n}]$ respectively. After we perform downsampling, according to nyquist's rule, we obtain approximation and detail coefficients. This process is called as one level decomposition. While doing the next level decomposition, already obtained approximation coefficients are passed through low pass and high pass filter and down sampled again, which means a two level decomposition. This number of decompositions are purely optional till we are able to get any abrupt notable variation in the detail coefficients while the short or open fault occurs. In this work we have taken a 11 level decompositions. In 11th level detail coefficients, we are able to catch the distinct changes with appreciable magnitudes while the faults occur. This process is done sequentially for all the phases of the stator coils. While we display the waveforms, the approximation coefficients will look similar to the original 1D signal and details coefficients contain only the high frequency components which depends on the level of the decomposition. The decomposition structure is shown in Fig. 5. 


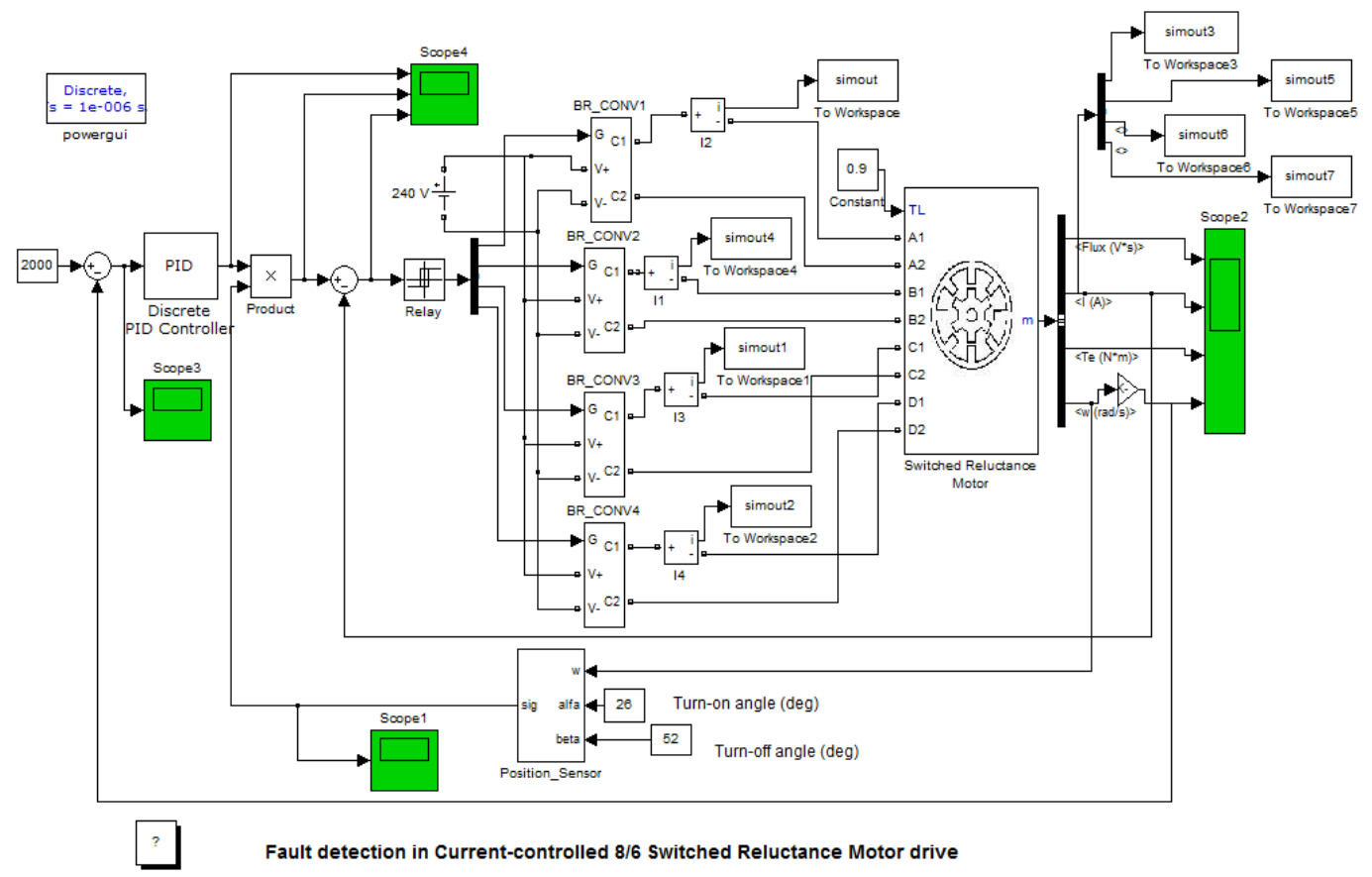

Fig. 4. Simulation model of $8 / 6 \mathrm{SRM}$

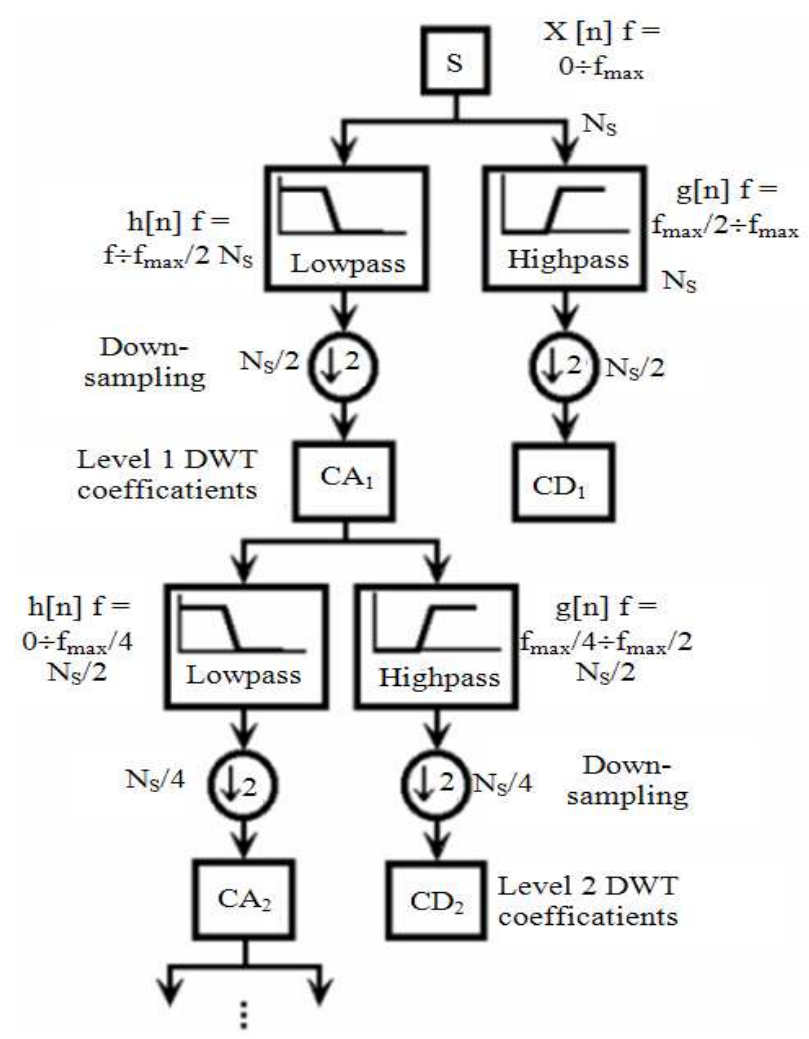

Fig. 5. Wavelet transform schematic 
The measured and saved values of the line currents were exported in MATLAB for further stage to classify to know which phase of the SRM is with short circuit or open circuit fault. As shown in Fig. 6-15, the obtained $\mathrm{d}_{11}$ wavelet coefficient's are plotted for normal and faulty SRM at a constant speed and constant load. From all the figures it is clear that, the magnitude of the detail coefficients are high exactly at the fault occurrence time.

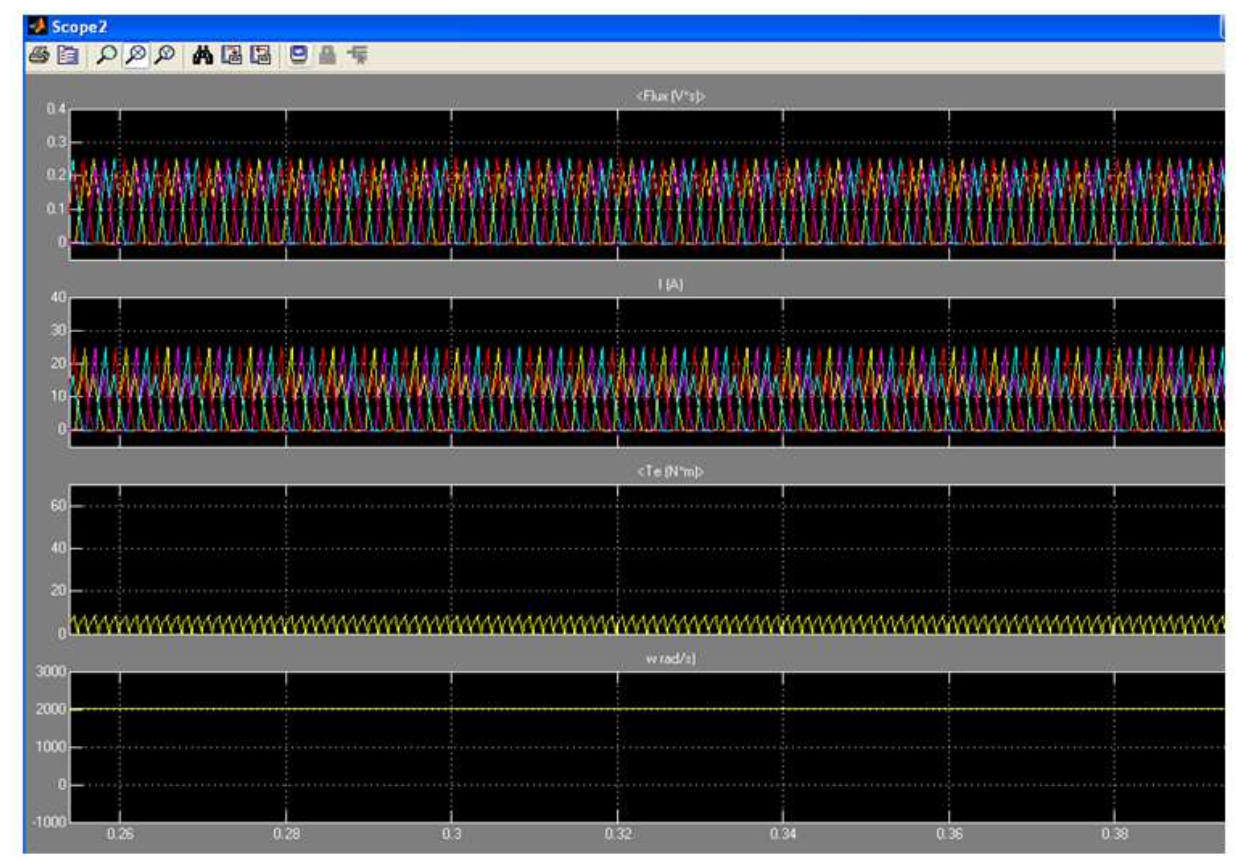

Fig. 6. Steady state waveforms of flux, current, torque and speed

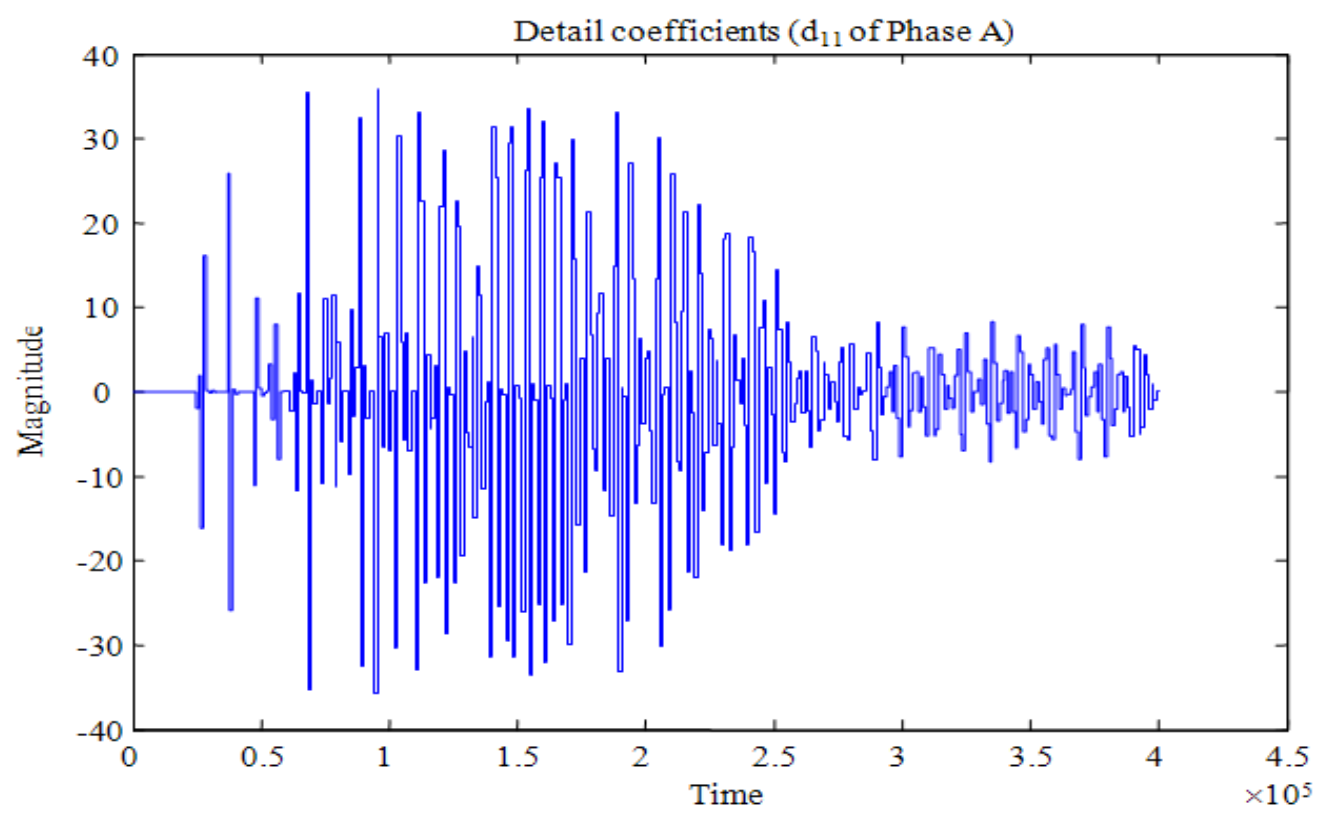

Fig. 7. Detail coefficient $\left(d_{11}\right)$ of phase A current 
Chandrika, V.S. and A. Ebenezer Jeyakumar / Journal of Computer Science 10 (5): 884-895, 2014

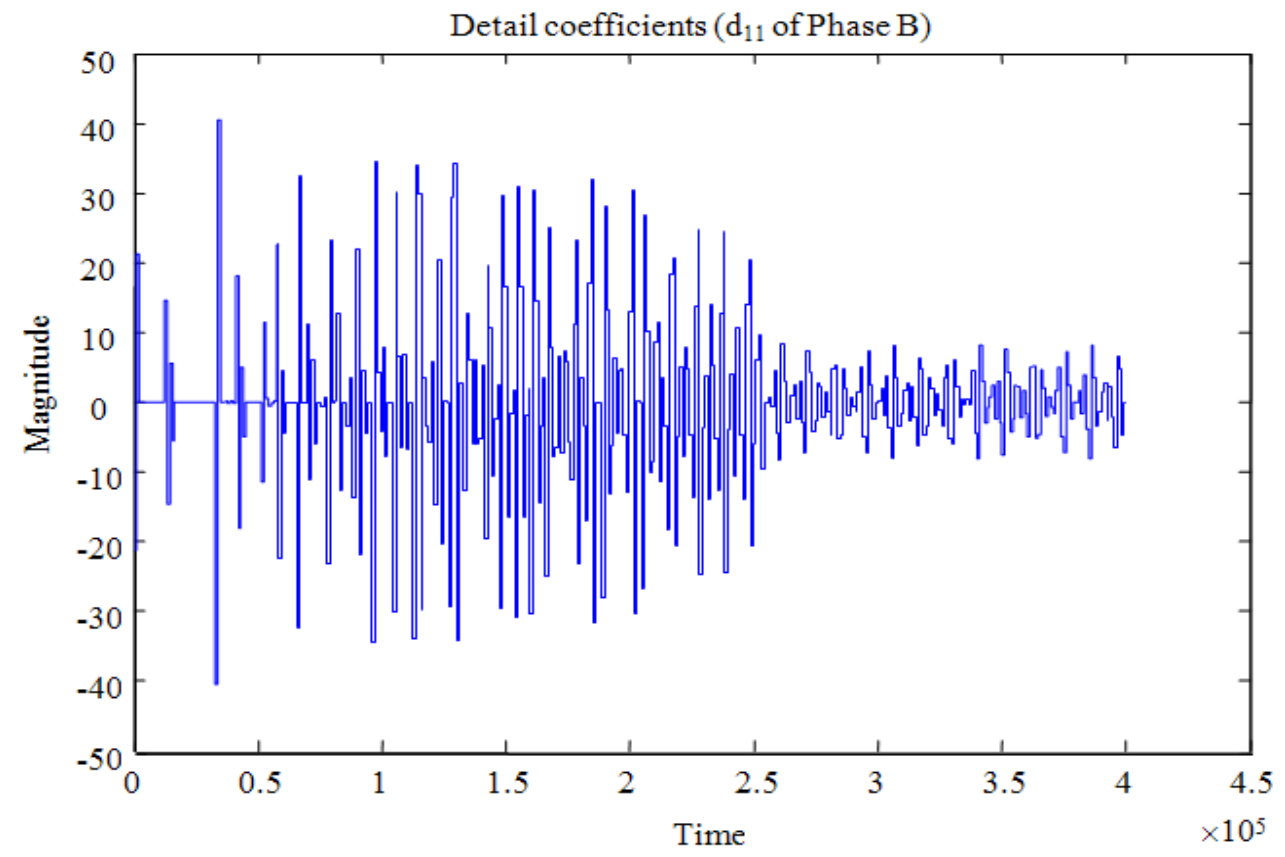

Fig. 8. Detail coefficient $\left(d_{11}\right)$ of phase B current

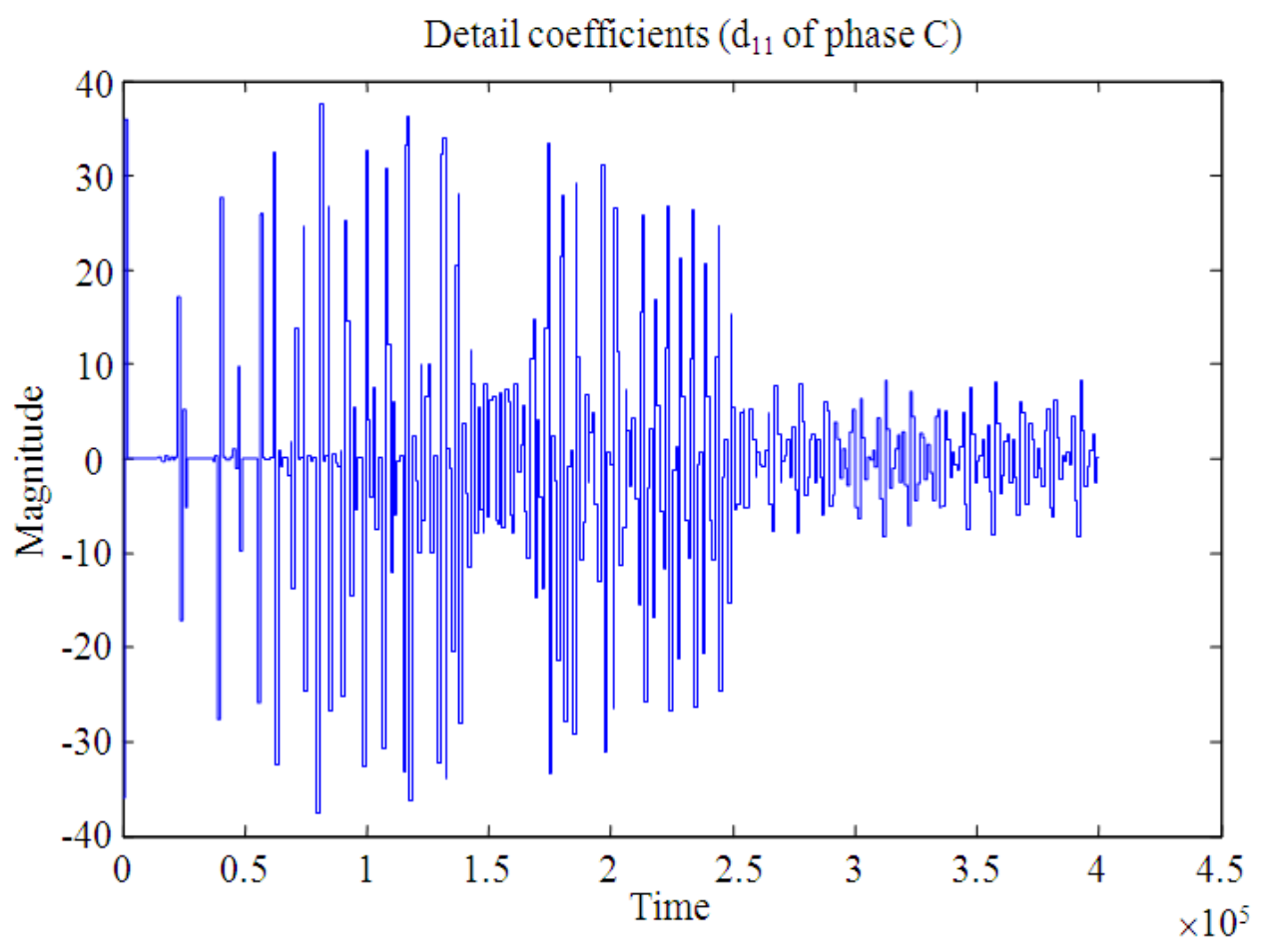

Fig. 9. Detail coefficient $\left(d_{11}\right)$ of phase $C$ current 
Chandrika, V.S. and A. Ebenezer Jeyakumar / Journal of Computer Science 10 (5): 884-895, 2014

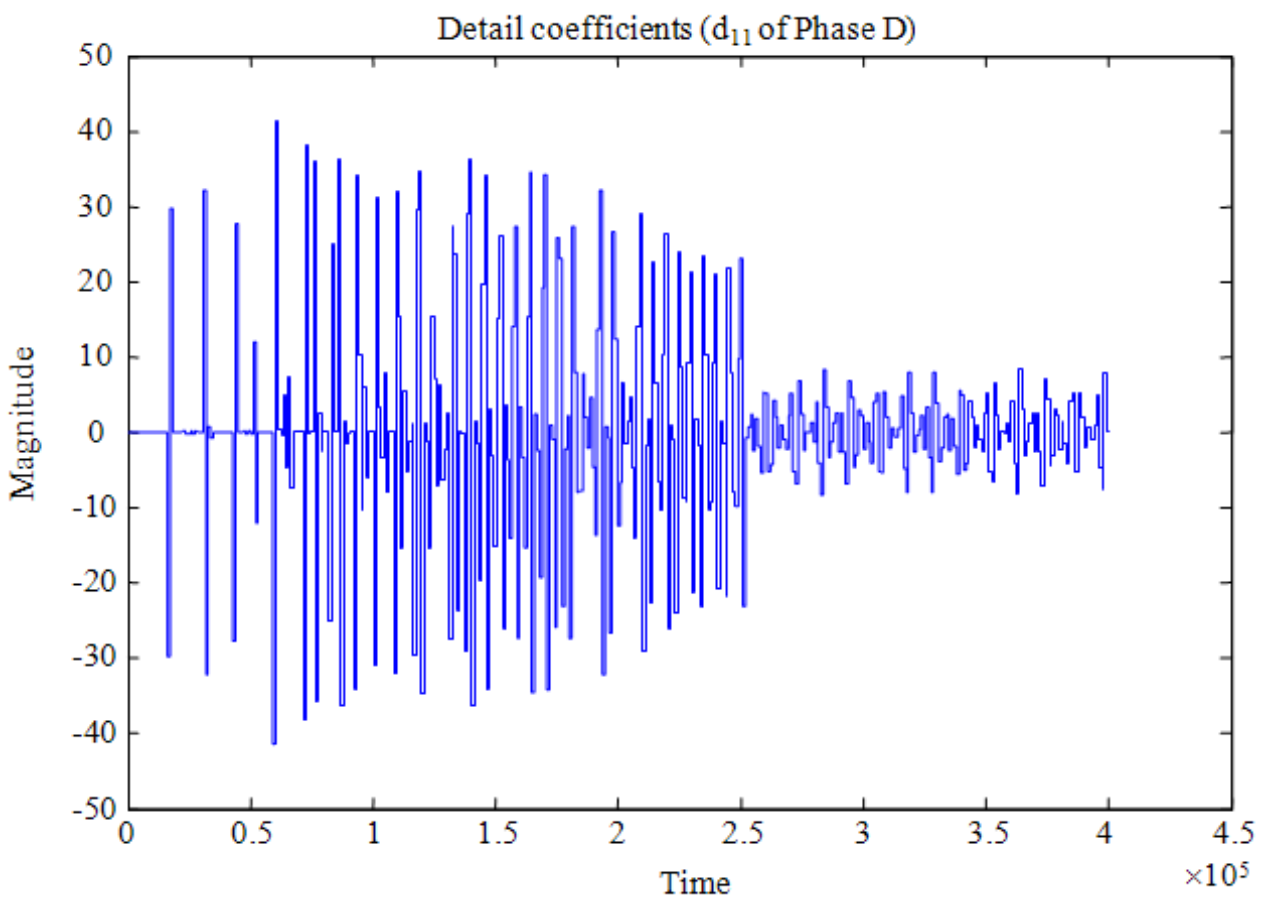

Fig. 10. Detail coefficient $\left(d_{11}\right)$ of phase $D$ current

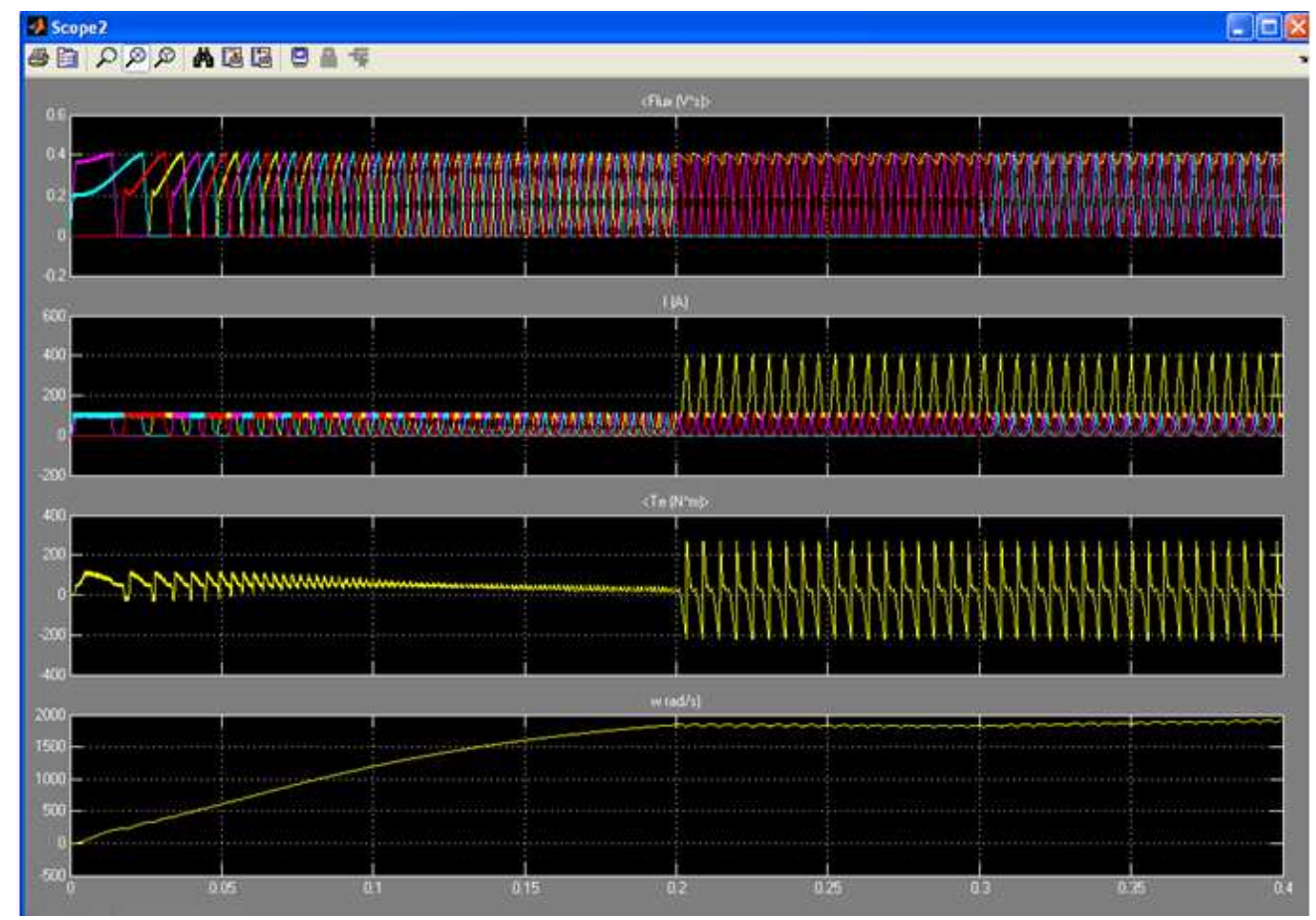

Fig. 11. Steady state waveforms of flux, current, torque and speed at phase a shorted and phase d opened 
Chandrika, V.S. and A. Ebenezer Jeyakumar / Journal of Computer Science 10 (5): 884-895, 2014

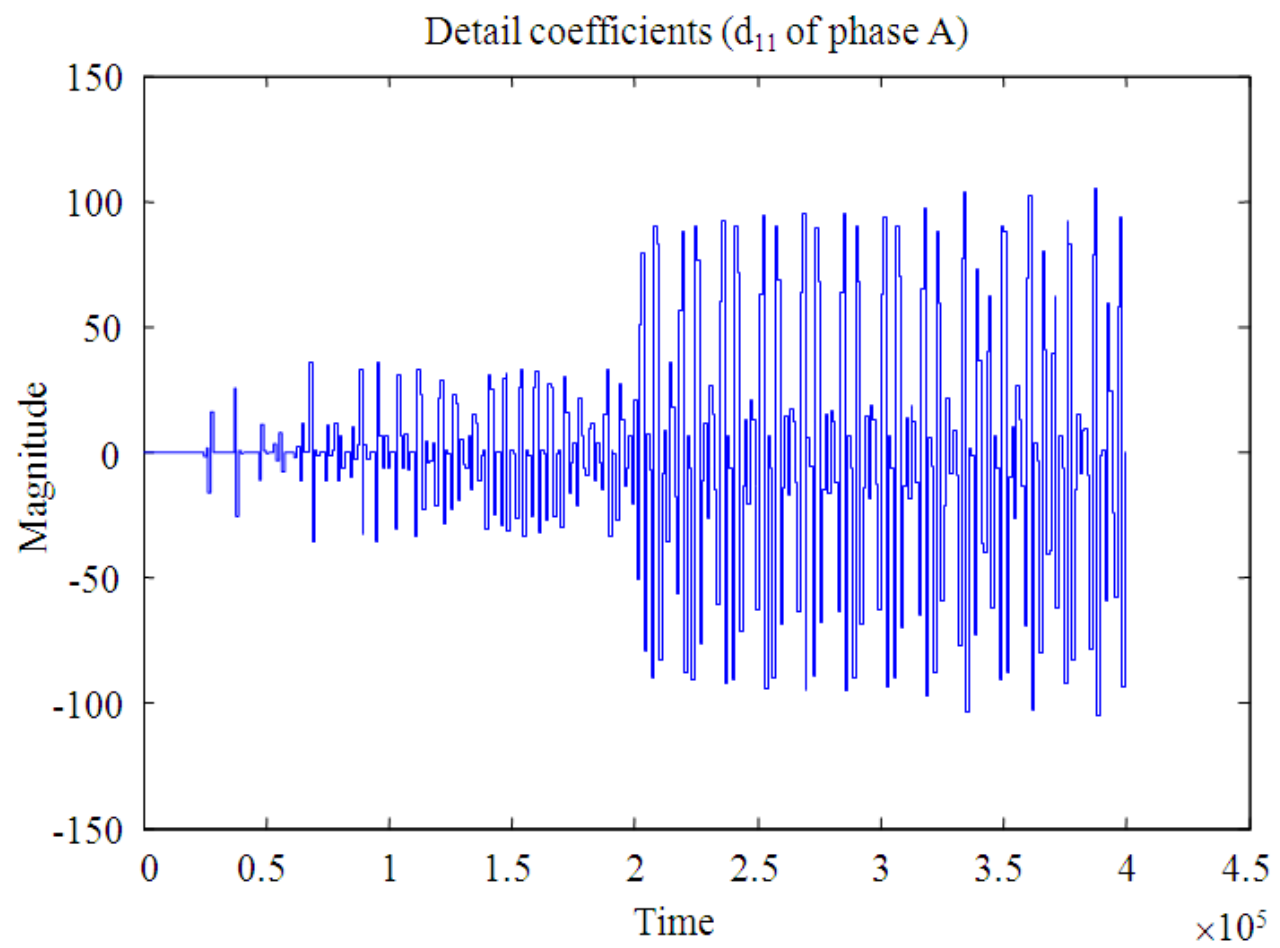

Fig. 12. Detail coefficient $\left(d_{11}\right)$ of phase A current

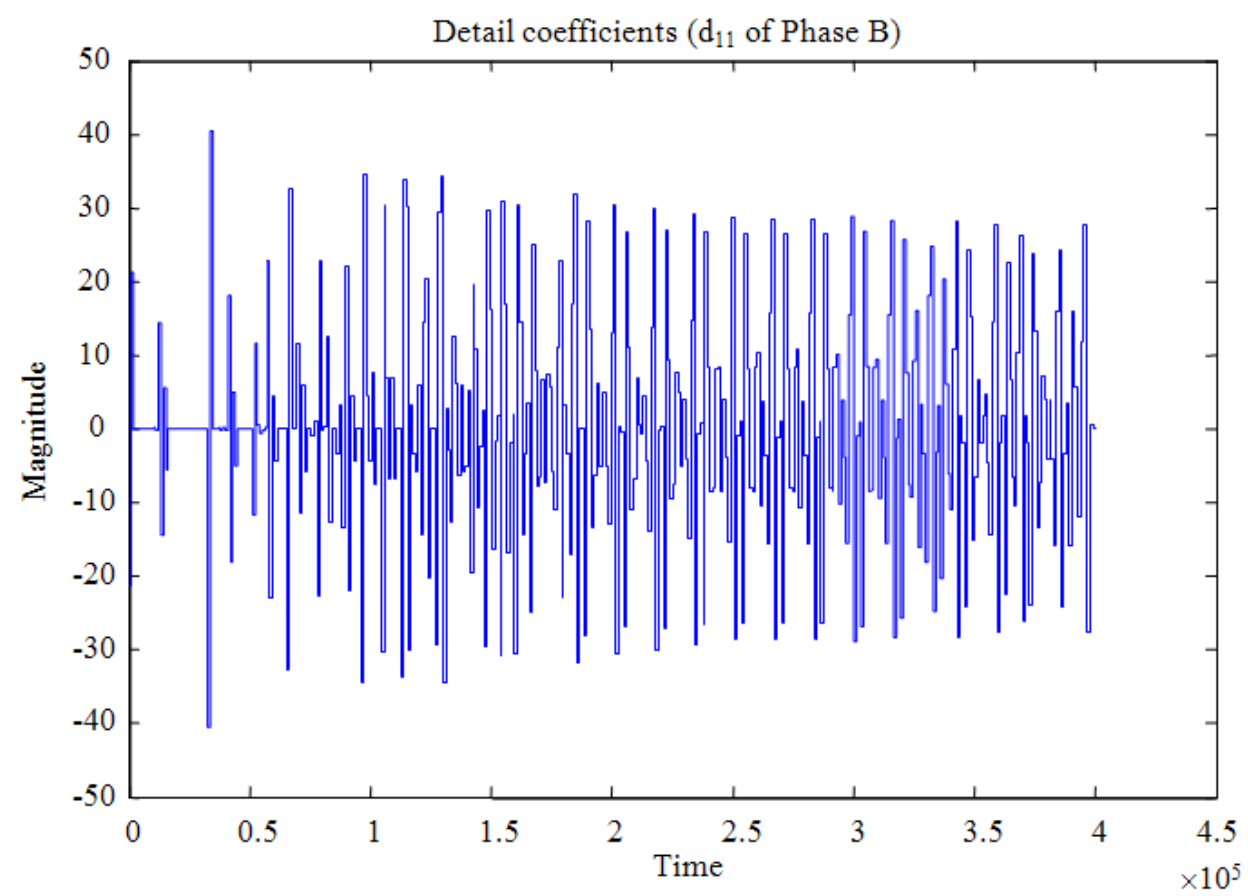

Fig. 13. Detail coefficient $\left(\mathrm{d}_{11}\right)$ of phase $B$ current 
Chandrika, V.S. and A. Ebenezer Jeyakumar / Journal of Computer Science 10 (5): 884-895, 2014

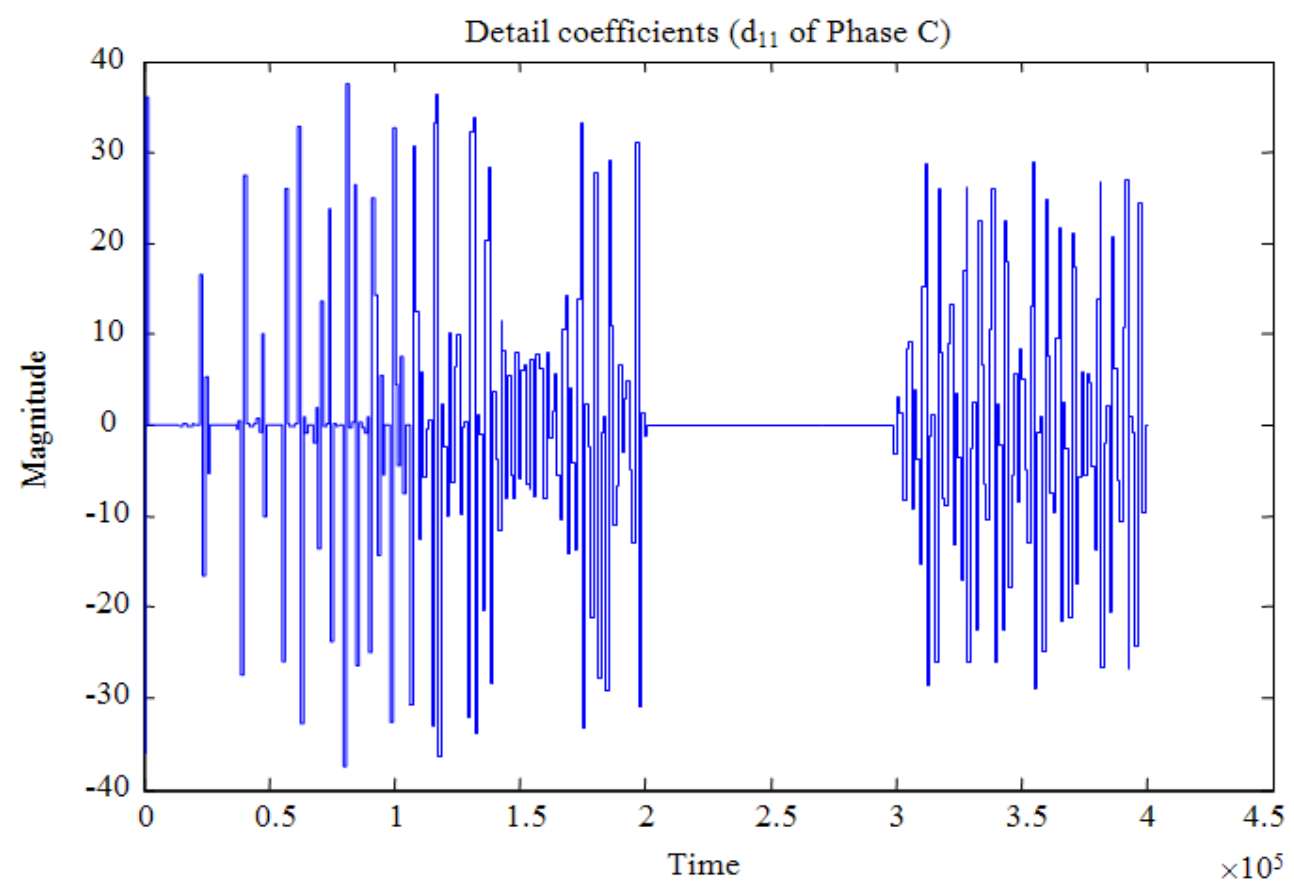

Fig. 14. Detail Coefficient $\left(d_{11}\right)$ of Phase C Current

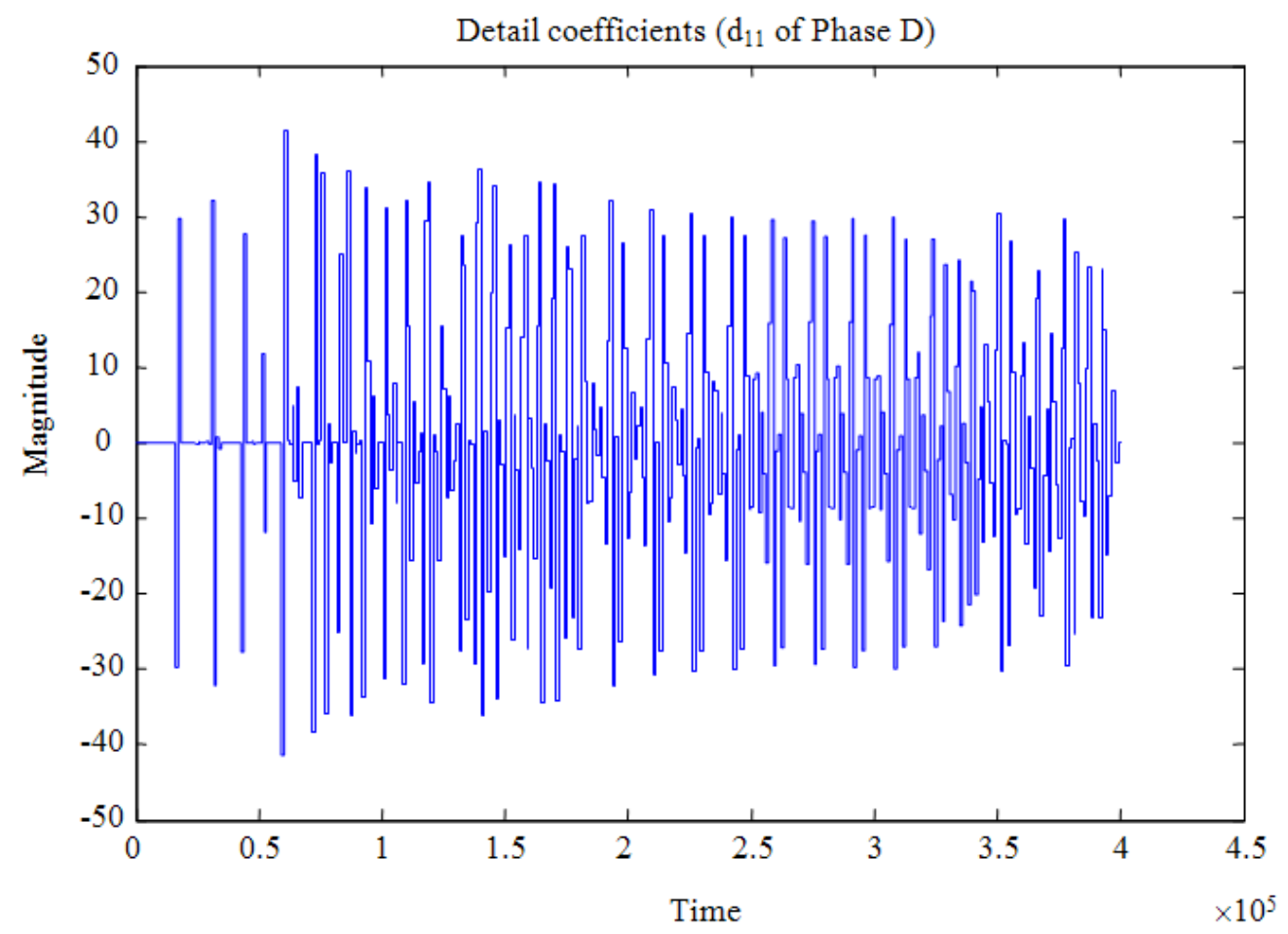

Fig. 15. Detail Coefficient $\left(d_{11}\right)$ of Phase D Current 


\section{SIMULATION OUTPUTS}

Figure 6 shows the output of the SRM drive with a speed of $2000 \mathrm{rpm}$. The parameters like, Flux, Stator current, Torque variations and Speed is shown. This is the output obtained at healthy conditions of the drive. Corresponding $\mathrm{d}_{11}$ coefficient has been shown in Fig. 7-10 shows the output of the SRM drive with a speed of $2000 \mathrm{rpm}$ under certain power switches short circuited exactly at time $\mathrm{t}=0.2 \mathrm{~s}$. The same parameters, Flux, Stator current, Torque variations and Speed is shown in Fig. 11. Corresponding $d_{11}$ coefficients have been shown in Fig. 12-15.

\subsection{Healthy Condition}

A Matlab simulink model has been designed and simulated with all the switches at perfect healthy conditions. The parameters have been shown at steady state. Load torque is set as 0.9 . Figure 6 shows only the steady state portion of the parameters. Whereas the Fig. 7-10 shows the values of $d_{11}$ coefficient from $t=0$.

Phase A and Phase C are short circuited and open circuited respectively at $\mathrm{t}=0.2 \mathrm{~s}$. It is seen from the Figure 11 that, the torque and the speed becomes oscillatory. More over, the flux of short circuited coils saturates to the maximum possible value. It is observed from Fig. 12 and 14, that the detail coefficient value exceeds a threshold 40 and reaches 100 and at open condition, the value rests at 0 . Also the time instant of the fault is found out as 0.2041 for phase A and 0.2058 for phase C. Further, the maximum values of detail coefficient is applied to classification algorithm to find the nature of fault in specific switches of the corresponding phases.

\section{CONCLUSION}

In this study, a DWT based method to detect the short circuit and open circuit fault and classification based on Back propagation network has been implemented. Also, time of fault occurrence has also been found out in this work. Hence, earlier detection of short circuit increases the life time of the SRM. This method also eliminates the use of any fuses or electronic circuitry to detect the faults. Simply capturing the waveform and applying sequentially to our algorithms is sufficient to detect and classify the fault. A sufficient level of intelligence has been added in the SRM drive through our work.

\section{REFERENCES}

Gameiro, N.S. and A.J.M. Cardoso, 2012. A new method for power converter fault diagnosis in SRM drives. IEEE Trans. Indus. Applic., 48: 653-662. DOI: 10.1109/TIA.2011.2180876

Jaganathan, B., R. Brindha ans S.K.S. Ait, 2011. Optimal parameters estimation of a switched reluctance motor by kohen's self organizing feature map method. IJCA Special Issue Artif. Intell. Techniques Novel Appr. Practical Techniques.

Karthikeyan, R., K. Vijayakumar and R. Arumugam, 2010. Study on swtiched reluctance generator for rural electrification. Int. J. Comput. Applic.

Mohanraj, K., S. Makkapati and S. Paramasivam, 2012. Unbalanced and double line to ground fault detection of three phase vsi fed induction motor drive using fuzzy logic apporach. Int. J. Comput. Applic.

Paramasivam, S. and R. Arumugam, 2004. Real time hybrid controller implementation for switched reluctance motor drive. Am. J. Applied Sci., 1: 284294. DOI: 10.3844/ajassp.2004.284.294

Salankayana, S.K., N. Chellamal and R. Gurram, 2012. Diagnosis of faults due to misfiring of switches of a cascaded h-bridge multi-level inverter using artificial neural networks. Int. J. Comput. Applic., 41: 17-22.

Shanmugam, N., A.B. Suryanarayana, T.S.B. Sudarshan, D. Chandrashekar and C.N. Manjunath, 2011. A novel approach to medical image segmentation. J. Comput. Sci., 7: 657-663. DOI: 10.3844/jcssp.2011.657.663

Sivakumar, M. and R.M.S. Parvathi, 2013. Particle swarm and neural network approach for fault clearing of multilevel inverters. Am. J. Applied Sci., 10: 579-595. DOI: 10.3844/ajassp.2013.579.595

Susitra, D., E. Annie, E. Jebaseeli and S. Paramasivam, 2010. Switched reluctance generator-modeling, design, simulation, analysis and control a comprehensive review. Int. J. Comput. Applic., 1: 10-16. DOI: $10.5120 / 90-189$ 